\title{
Promovendo a autoridade e o poder da gestante: uma atividade da enfermagem na construção da cidadania*
}

\author{
Juliana Vieira Figueiredo' \\ Lydia Vieira Freitas ${ }^{2}$ \\ Thais Marques Lima ${ }^{3}$ \\ Amanda Souza de Oliveira ${ }^{4}$ \\ Ana Kelve de Castro Damasceno ${ }^{5}$
}

Aceito para publicação em setembro de 2010

\begin{abstract}
Objetivou-se descrever uma experiência educativa realizada com gestantes da rede pública de saúde de Fortaleza. Estudo descritivo, efetuado no Laboratório de Comunicação (Labcom) do Departamento de Enfermagem da Universidade Federal do Ceará, de agosto a novembro de 2008. A atividade educativa teve seu conteúdo dividido em três módulos baseados em estratégias participativas, que visavam a possibilitar a elevação do conhecimento das gestantes com relação ao trabalho de parto e ao parto propriamente dito. A amostra foi composta de oito gestantes, que estiveram presentes no dia em que os aspectos relacionados ao trabalho de parto foram estudados. Após o desenvolvimento da estratégia, as gestantes relataram a importância da realização de atividades educativas para esse período de sua vida. Concluímos que a educação em saúde é uma importante estratégia para a aquisição de conhecimentos para as gestantes, proporcionando-lhes o desenvolvimento da autoridade com relação ao autocuidado e ao cuidado com seu filho, bem como uma atividade fortalecedora para a enfermagem.
\end{abstract}

Descritores: Gestantes, Educação em Saúde, Enfermagem.

\section{Promoting the authority and the power of the pregnant woman: a nursing activity in the construction of citizenship}

The aim of this paper was to describe an educational experience with pregnant women from Fortaleza public health. This descriptive study was done at the Laboratory of Communication (LABCOM) Department of Nursing, Federal University of Ceará, from August to November 2008. The educational activity was divided into three content modules based on participatory strategies, aimed at facilitating the increase of knowledge of pregnant women regarding labor and delivery itself. The sample consisted of eight pregnant women, who were present on the day that the aspects related to labor were addressed. After developing the strategy, the women reported the importance of educational activities for this period of their lives. We concluded that health education is an important strategy for the acquisition of knowledge to women, providing them the development of authority in relation to their care and care for her child as well as an empowering activity for nursing.

Descriptors: Pregnant Women, Health Education, Nursing.

\section{Promoción de la autoridad y el poder de la mujer embarazada: una actividad de enfermería en la construcción de la ciudadanía}

El objetivo fue describir una experiencia educativa con mujeres embarazadas en la salud pública de Fortaleza. Se trata de un estudio descriptivo realizado en el Laboratorio de la Comunicación (LabCom) Departamento de Enfermería de la Universidad Federal de Ceará, entre agosto y noviembre de 2008. La actividad educativa se dividió en tres módulos de contenidos basados en estrategias participativas, dirigidas a facilitar el aumento de los conocimientos de las mujeres embarazadas en relación con el parto en sí. La muestra fue compuesta por ocho mujeres embarazadas, que estaban presentes el día en que los aspectos relacionados con el trabajo de parto se abordaron. Después de desarrollar la estrategia, las mujeres informaron la importancia de las actividades educativas para este período de su vida. Concluimos que la educación de la salud es una estrategia importante para la adquisición de conocimientos a las mujeres embarazadas, proporcionándoles el desarrollo de la autoridad en relación a su cuidado y atención para su hijo, así como potenciar la actividad de enfermería.

Descritores: Embarazo, Educación para la Salud, Enfermería.

\section{INTRODUÇÃO}

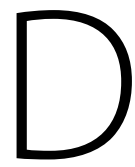

urante a gestação, diversas mudanças ocorrem no organismo da mulher, que necessita de inúmeras informações sobre o ciclo gravídico-puerperal, que Ihe permitirão a aquisição de poder de decisão e autonomia para cuidar de si e do filho, conquistando autoridade para lidar com tais mudanças.

Por meio da conquista da autonomia, a mulher desenvolve o direito de cidadania, contido na Constituição de 1988, que é

1 Discente do curso de enfermagem da Universidade Federal do Ceará (UFC). Bolsista do Programa de Educação Tutorial (PET Enfermagem/MEC - Sesu).

2 Enfermeira. Mestranda em enfermagem pela Universidade Federal do Ceará (UFC). Bolsista CNPq.

3 Enfermeira. Mestranda em enfermagem pela Universidade Federal do Ceará (UFC). Bolsista Capes.

4 Discente do curso de enfermagem da Universidade Federal do Ceará (UFC). Bolsista CNPq.

5 Enfermeira. Especialista em enfermagem obstétrica. Docente adjunta III da UFC. Tutora do Programa de Educação Tutorial (PET Enfermagem/MEC - Sesu).

* Apoio financeiro da Fundação Cearense de Apoio ao Desenvolvimento Científico e Tenológico - Funcap. 
compreendida como o exercício da plenitude dos direitos ${ }^{(1)}$. A descoberta da gestação representa um importante momento para a mulher, o parceiro e a família, acarretando uma série de sentimentos que tornam esse momento peculiar. Os profissionais de saúde assumem uma posição de apoiadores e participantes desse processo, pois constituem fonte de informações para as gestantes durante esse ciclo.

Como profissional atuante no cuidado à saúde, o enfermeiro desenvolve atividades de educação que promovem a autonomia da gestante, permitindo que ela compreenda as modificações existentes no organismo e como lidar com as mesmas, fornecendo-lhe e permitindo o direito à cidadania. Dessa forma, esse profissional está exercendo a cidadania, visto que realiza o dever profissional e promove o direito da gestante.

No primeiro contato do enfermeiro com a gestante, faz-se necessário conhecer o passado e o presente obstétrico e iniciar uma relação de escuta e confiança, de forma a preparar a mulher para receber e colocar em prática as orientações necessárias para a saúde dela e do bebê $\hat{e}^{(2)}$.

Essas orientações fornecidas durante o pré-natal são de suma importância, já que preparam a mulher para o período gestacional, o trabalho de parto e os cuidados com si mesma e com o neonato. Durante o trabalho de parto e seu desfecho, a presença dessas orientações se tornam evidentes, visto que deixam a mulher segura e confiante durante esse processo. Essas orientações favorecem a mulher a adquirir autoridade sobre o trabalho de parto.

Portanto, a participação ativa da mulher no trabalho de parto é fundamental, devendo opinar quanto ao local de parto, às diferentes modalidades de atenção na assistência, posição e método para o cuidado e conforto nos momentos que antecedem o nascimento, facilitando, assim, a evolução do parto ${ }^{(3)}$.

Sob tal perspectiva, entendemos que a educação em saúde é mais do que a imposição de valores por parte dos profissionais para o público, e sim percebida como relação horizontal entre esses, na qual os profissionais são mediadores da saúde, assumindo uma educação não condutivista, em que os indivíduos realizam escolhas dentro do respeito à liberdade ${ }^{(4)}$.

Quanto às parturientes, quando não possuem autonomia em saúde, se submetem às ordens dos profissionais que estão ao redor, sem questionar. Com isso, essas mulheres deixam de ser as protagonistas do processo de parturição, passando à condição de expectadoras do próprio viver, postura resultante de uma assistência pré-natal munida de poucas informações.

Visando a combater essa situação de completa inatividade por parte da mulher com relação ao ciclo gravídico-puerperal, nasceram na Europa, no século 20, pelas mãos de Dick Read, obstetra inglês, os cursos de preparação para o parto, com os objetivos de: prover a gestante de informações necessárias sobre gravidez, parto e cuidados ao recém-nascido; vencer a ansiedade inerente ao parto; reduzir a dor com técnicas de respiração e relaxamento; despertar na mulher processos de aprendizagens e conhecimentos de seu corpo, de modo que venha a desempenhar papel pró-ativo e positivo de cuidado da saúde; proporcionar o encontro com outras mulheres em situação semelhante; orientar o futuro pai para que esteja o mais próximo possível da mulher; apresentar à mulher o ambiente do processo de parturição ${ }^{(5)}$.

Vale ressaltar que, quando os indivíduos estão em atividades grupais, conseguem produzir modificações de comportamento a partir da reciprocidade de relações e entrelaçamentos de atos, concepções e sentimentos e desenvolvem o comprometimento dos indivíduos com a saúde ${ }^{(6)}$. Com essas informações, vê-se a importância de ações, dada a possibilidade de fornecer um processo de parturição mais tranquilo por parte das gestantes, para que abandonem sua comum e atual postura submissa em sala de parto, tornado-as capazes de tomar as decisões nesse momento, bem como ao longo da assistência pré-natal ofertada.

Diante do exposto, temos como objetivo descrever a experiência de uma estratégia educativa realizada com gestantes atendidas na rede pública de saúde.

\section{METODOLOGIA}

Estudodenaturezadescritiva, quesecaracteriza por ser flexível e holístico, permitindo a adequação de novas informações ao longo da implementação das atividades, visa a entender a totalidade dos aspectos analisados e requer, portanto, um intenso envolvimento do profissional nodesenvolvimentodapesquisa ${ }^{(7)}$. O estudo se baseou em um dos tópicos trabalhados com o grupo de gestantes durante a estratégia educativa, denominada por pesquisadoras e participantes de curso de gestantes.

O curso consistiu em um conjunto de atividades educativas sobre o ciclo gravídico-puerperal realizado de agosto a novembro de 2008, tendo temáticas divididas em três módulos: Módulo I: Conhecendo as modificações corporais da gravidez, aspectos relacionados a assistência pré-natal; Módulo II: Preparando para o aleitamento materno e cuidados com o recém-nascido; e Módulo III: Preparando a gestante para o trabalho de parto, o parto e o puerpério. As atividades foram distribuídas em 11 momentos, que ocorriam semanalmente, durando, cada um, em torno de uma hora e meia. O conteúdo do curso está de acordo com a figura 1.

Destacam-se no presente trabalho as estratégias que visavam a possibilitar a elevação do conhecimento das gestantes com relação ao momento do trabalho de parto e o parto.

O estudo foi composto de gestantes que realizavam o prénatal em uma Unidade Básica de Saúde e em uma maternidade de referência. Essas mulheres foram convidadas a comparecer ao Laboratório de Comunicação (Labcom) do Departamento de Enfermagem (Denf) da Universidade Federal do Ceará (UFC), que se constituiu no local de realização do curso de gestantes. As participantes foram oito gestantes que aceitaram integrar o estudo e que compareceram ao curso no dia em que os aspectos relacionados ao trabalho de parto foram estudados 


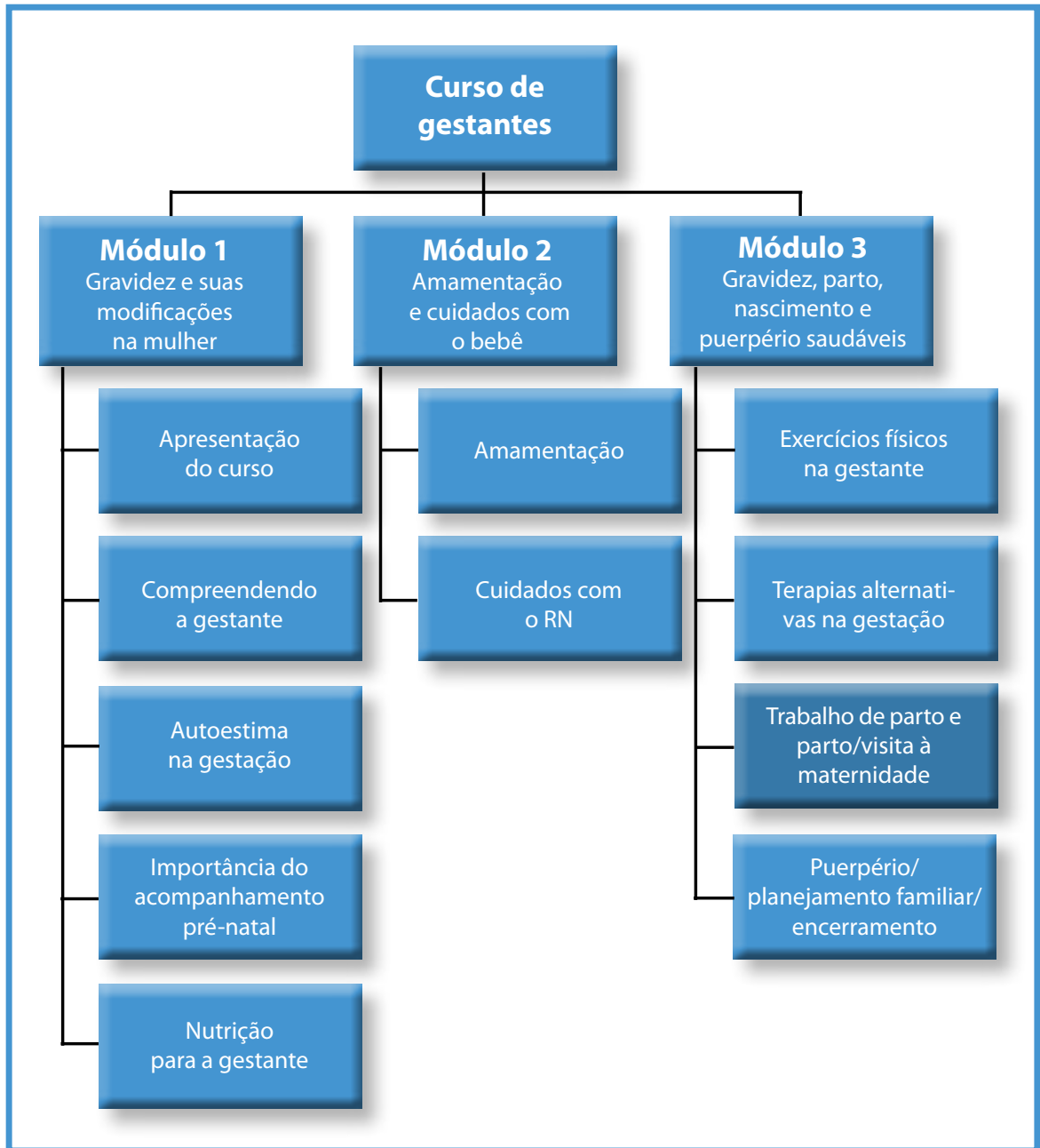

Figura 1 - Conteúdo programático do curso de gestantes, Denf-UFC, 2008

entre o grupo. Essa primeira turma do curso de gestantes tornou-se um projeto piloto para estruturar os módulos e assim possibilitar uma oferta anual. Por se configurar como projeto piloto, o tamanho do grupo a ser observado foi limitado.

Participaram desse grupo tanto primigestas quanto multigestas, constituindo assim um grupo misto, tendo em vista a relevância do compartilhamento de informações e experiências entre as gestantes, de forma que as primigestas possam ouvir as vivências das multigestas, sendo a recíproca igualmente válida.

O estudo ocorreu em duas etapas: 1) atividade educativa, que tinha por objetivo dialogar com as mulheres sobre o processo de parturição; 2) realização da visita à maternidade na qual as mulheres pretendiam vivenciar o processo de parturição.

Durante a atividade, foi utilizado como um dos instrumentos de coleta de dados o diário de campo, por meio do qual as pesquisadoras puderam observare registraros comportamentos apresentados pelas gestantes durante a atividade educativa. A coleta de dados baseada em diário de campo consiste em uma técnica utilizada em pesquisas científicas e atividades acadêmicas, que permite o registro de observações e vivências em prol do avanço em diferentes áreas ${ }^{(8)}$.
Além do diário de campo, aplicamos um instrumento, antes da atividade educativa, de quatro perguntas pertinentes ao tema, abordando indicação da via de parto, técnicas de relaxamento na gravidez que trazem melhorias no momento do parto e respiração, o direitoà licença-maternidade e cuidados com o corpo no puerpério imediato. Um segundo instrumento que visou a verificar o conhecimento adquirido pelas gestantes, aplicado com o grupo após a estratégia, contava com três questões sobre a temática citada.

$O$ projeto referente a esse estudo foi aprovado pelo Comitê de Ética em Pesquisa (Comepe) da UFC.

\section{RESULTADOS E DISCUSSÃO}

Em virtude da experiência profissional das pesquisadoras, que reconheciam a síndrome do trabalho de parto como tema repleto de dúvidas por partedas gestantes, e conscientes do fato de que muitas vezes os companheiros compartilhavam essas dúvidas, elas estenderam a eles o convite para que comparecessem. Contaram com a presença de três companheiros, o que causou entusiasmo por parte das gestantes acompanhadas. Entretanto, as demais apresentavam certa frustração pela ausência do companheiro.

Após a aplicação do primeiro instrumento, observou-se que as gestantes participantes acertaram todos os quesitos, com exceção das técnicas de relaxamento. A realização de técnicas de relaxamento durante o período gestacional tem por objetivo conferir tranquilidade durante o trabalho de parto e o parto. Isso nos mostra o quanto algumas práticas complementares de saúde estão no descrédito de parte da população.

Diversas técnicas de relaxamento podem ser utilizadas pela mulher durante a gestação e durante o trabalho de parto e o parto. Elas incluem massagens nos pés e nas pernas, meditação, sono, exercícios de respiração e relaxamento, alimentos que proporcionam tranquilidade, shiatsu e ioga, entre outras. A partir do conhecimento dessas atividades, a mulher adquire poder para promover o autorrelaxamento, conquistando autonomia para a promoção de seu bem-estar.

Um ensaio clínico foi realizado com 100 parturientes em uma maternidade pública de Natal (RN) sobre o tipo de intervenção terapêutica para avaliar a efetividade de técnicas não farmacológicas de relaxamento para o alívio da dor de parturientes durante o parto. Foram aplicados exercícios respiratórios, relaxamento muscular, massagem lombossacral e banho de chuveiro, concluindo-se que as estratégias foram efetivas no alívio da intensidade da dor ${ }^{(9)}$. 
Realizamos uma aula expositiva sobre o trabalho de parto e o parto, utilizando como recurso visual o equipamento de multimídia para a melhor compreensão das mulheres a respeito do assunto, tentando discutir com elas as principais dúvidas sobre esses tópicos. Nesse momento, a maioria demonstrou dúvidas sobre o momento certo de ir para a maternidade, relatando medo de sentir algo e não saber o que fazer, medo esse também explicitado por parte do companheiro. Essa situação pode desencadear o processo de peregrinação durante o trabalho de parto, caracterizado por diversas idas e vindas à maternidade, até a mulher estar pronta para ser admitida no serviço hospitalar.

Estudo com os frequentadores de um curso de orientação à gestação na cidade de São Paulo verificou que os participantes procuraram o curso em busca de conhecimento e esclarecimento de dúvidas do parto e cuidados com a gestante e com o recém-nascido ${ }^{(10)}$.

O exercício da autonomia da parturiente requer que a mesma tenha alguns conhecimentos para que possa exercêla com maior efetividade, tornando-a ativa nesse processo. Porém, isso não deve ser limitado à pessoa, mas sim compartilhado com os outros membros da família, sendo o companheiro nesse período o principal apoio; contudo, isso não significa que o mesmo esteja preparado para esse momento.

Outra ansiedade demonstrada pelas gestantes era relativa à necessidade (ou possibilidade) do acompanhante na sala de parto. Algumas dessas mulheres conheciam esse direito, relatando inclusive que gostariam de passar pelo processo de parturição no serviço visitado posteriormente, por conhecerem a garantia da presença de um acompanhante durante o trabalho de parto e o parto.

Com o surgimento da lei do acompanhante por meio da portaria interministerial de 2005, a parturiente em trabalho de parto, parto e puerpério tem assegurada a presença de um acompanhante de escolha. Na prática, esse acompanhante não está preparado para apoiar a mulher nesse momento em que laços familiares são importantes para a determinação do processo de parturição tranquilo. É durante atividades de educação em saúde realizadas na assistência pré-natal que os profissionais de saúde devem incentivar a participação do companheiro ou familiar nesse atendimento, para realizar orientações acerca do trabalho de parto, parto e puerpério e de como poderão ajudar ${ }^{(11)}$.

Estudo realizado em maternidade de Londrina (PR) com o objetivo de apreender o conhecimento dos pais sobre o direito do acompanhante durante o trabalho de parto e parto e conhecer a vivência deles durante o nascimento dos filhos verificou que eles desconheciam o direito que lhes é resguardado por lei de estarem presentes, atribuindo sua presença à benevolência da equipe médica ${ }^{(12)}$. Ao oferecer informações ao companheiro, o mesmo adquire poder para tranquilizar a gestante e orientá-la de maneira adequada sobre o processo do parto, reduzindo a ansiedade de ambos.

Ressalta-se a pergunta relativa à necessidade de realização de episiotomia, chamada pelas mulheres de "corte". As gestantes questionaram se esse procedimento era rotineiro, ao que foi explicado que dependeria da necessidade da parturiente, sendo comum em primíparas.

Estudo realizado em um centro de referência de Recife (PE) com mulheres submetidas a parto normal, no qual se objetivou avaliar a prevalência e fatores associados à realização de episiotomia, verificou uma prevalência de $29 \%$, e os fatores associados foram doenças maternas e ausência de parto vaginal anterior ${ }^{(13)}$.

Tentou-se frisar a identificação da síndrome do trabalho de parto pela gestante, que consiste em contrações dolorosas, rítmicas (no mínimo duas em dez minutos), que se estendem a todo o útero com duração de 50 a 60 segundos; colo apagado nas primíparas e dilatado para 2 centímetros; nas multíparas, semiapagado e com 3 centímetros de dilatação; rompimento da bolsa de águas; e perda do tampão mucoso ${ }^{(14)}$

A explicação ocorreu de maneira didática, chamando a atenção para o que poderiamser (contrações, perda dotampão e de líquido amniótico) e o que, ao chegar à maternidade, será visto pelo profissional de saúde durante o exame obstétrico, no momento do toque vaginal (dilatação e apagamento), fazendo-se analogia para que haja melhor compreensão.

Observa-se muitas vezes o despreparo das gestantes sobre a fisiologia em um trabalho de parto normal. Esse fato interfere na forma como os profissionais irão prestar assistência, pois, a partir do conhecimento sobre o que acontece com o corpo, a mulher consegue assumir postura e calma nesse processo, agindo de forma a fazer com que o trabalho de parto ocorra da melhor forma possível. Ter conhecimento por parte das usuárias é relevante, mas poderá ser condição ameaçadora ao poder e autoridade dos profissionais no cenário hospitalar.

Esse fato pode ser confirmado por meio de um estudo qualitativo realizado em uma maternidade de referência no Ceará, em 2008, sobre a presença do acompanhante em sala de parto. Na perspectiva dos profissionais de enfermagem, observou-se que a presença do acompanhante no cenário do parto provocou mudanças na postura dos profissionais frente à parturiente e à assistência prestada. $\mathrm{O}$ estudo ressalta que é necessário que a equipe convide o acompanhante a se envolver de forma ativa no processo de parturição( ${ }^{(15)}$.

Após esse momento educativo, as gestantes responderam individualmente a um segundo instrumento com os mesmos pontos do primeiro, de forma a possibilitar a comparação dos resultados, queabordou os temas: aumento do risco de infecção no parto cesáreo, direito à licença-maternidade e presença do pai durante o ciclo gravídico puerperal. As participantes do estudo foram unânimes em responder corretamente aos 
questionamentos, tendo o conhecimento sobre os riscos do parto cesáreo, o direito à licença-maternidade de seis meses para funcionárias públicas e quatro meses para o setor privado, bem como a licença-paternidade de sete dias para aqueles que se encontram no mercado formal de trabalho.

Após essa exposição dialogada, nós nos dirigimos para o serviço que seria visitado pelo grupo. De início, visitamos o setor de emergência da maternidade, por onde a mulher é admitida, ressaltando a importância de a gestante portar o Cartão de Pré-Natal. Informamos que, na admissão, seriam denominadas de parturiente, ou seja, gestante em trabalho de parto. Depois seguimos ao Banco de Leite Humano da maternidade, reforçando os inúmeros benefícios do aleitamento materno exclusivo e complementado. Na sequência, mostramos a entrada do Centro de Parto Humanizado e a entrada do Centro Cirúrgico. As mulheres foram informadas de que, havendo a necessidade de algum procedimento de maior complexidade, existe uma equipe permanente para esse fim, além da Unidade de Terapia Intensiva Neonatal. Reforçou-se que, durante o parto normal, as intercorrências são mínimas, mas existe a possibilidade de o bebê necessitar de um suporte tecnológico nas primeiras horas de vida.

Contamos com o apoio da instituição no que diz respeito a reservar uma enfermaria do Centro de Parto Humanizado para que pudessem conhecer o ambiente de parturição. Ainda tivemos a oportunidade de mostrar a enfermaria individualizada com leito próprio para a realização do parto normal, banheiro estruturado com barras de apoio, poltrona para acompanhante, bola de ginástica, cavalinho e barras de madeira para alongamento, assim como outros mobiliários como mesa de apoio, mesa de mayo, local para trocar o bebê e armários.
O objetivo da visita não era que as gestantes assistissem a um parto, até porque é um evento que pode causar ansiedade. Contudo, durante a visita, foi presenciado o parto de uma gestante multípara, pois a enfermeira obstetra que acompanhava o grupo precisou ajudar. A experiência foi positiva, visto que, de longe, era um cenário tranquilo quando a criança nasceu e quase todo o grupo viu. Isso representou um fator encorajador para as gestantes, comprovado pela fala de uma delas: "Ah, é tão rápido assim, eu já queria ter parto normal, agora é que eu vou ter mesmo".

Não poderia haver um desfecho melhor para a atividade de preparação do parto do que esse. Os objetivos de tornar a gravidez um momento de autonomia e fortalecimento da mulher por meio do curso de gestantes foram alcançados.

\section{CONSIDERAÇÕES FINAIS}

Pode-se inferir que o curso é estratégia para a aquisição de conhecimentos básicos à gestante, o que lhe proporciona o desenvolvimento da cidadania. Assim, a aquisição de poder/ autoridade será consequência desse processo de busca de saber, e a resposta imediata será o melhor atendimento por parte dos profissionais de saúde. No âmbito da parturição, esse processo educativo realizado previamente torna a mulher mais segura do que está vivenciando. Ela, assim, se torna um sujeito ativo, rebatendo a posição passiva, muitas vezes assumida devido a sentimentos de medo e insegurança.

Ressalta-se a relevância do companheiro, que se encontra em um nível secundário de prioridade educativa no que diz respeito ao ciclo gravídico-puerperal. Ele deveria ser mais bem trabalhado para fornecer à gestante, parturiente ou puérpera apoio maior nessa fase.

\section{Referências}

1. Pessini L, Barchifontaine CP. Problemas atuais de Bioética. $8^{\mathrm{a}}$ ed. São Paulo:

Loyola; 2007.

2. Couto GR. Conceitualização pelas enfermeiras de preparação para o parto.

Rev Latinoam Enferm. 2006;14(2):190-8.

3. Carraro TE, Knobel R, Frello AT, Gregório VRP, Grüdtner DI, Radünz V et al.

O papel da equipe de saúde no cuidado e conforto no trabalho de parto e parto:

opiniäo de puérperas. Texto Contexto Enferm. 2008;17(3):502-9.

4. Silva CMC, Meneghim MC, Pereira AC, Mialhe FL. Educação em saúde:

uma reflexão histórica de suas práticas. Ciênc Saúde Coletiva. 2010;15(5):2539-50.

5. Couto GR. Conceitualização pelas enfermeiras de preparação para o parto.

Rev Latinoam Enferm. 2006;14(2):190-8.

6. Silva JLA, Lopes MJM. Educação em saúde a portadores de úlcera varicosa

através de atividades de grupo. Rev Gaúcha Enferm. 2006;27(2):240-50.

7. Polit DF, Beck CT, Hungler BP. Fundamentos de pesquisa em enfermagem. 5a ed.

Porto Alegre: Editora Artmed; 2004.

8. Dal Pai D, Lautert L. Grupos de discussão virtual: uma proposta para o ensino em

enfermagem. Rev Esc Enferm USP. 2007;41(3):518-25.

9. Davim RMB, Torres GV, Dantas JC. Efetividade de estratégias não farmacológicas no alívio da dor de parturientes no trabalho de parto. Rev Esc Enferm USP.

2009;43(2):438-45.

10. Santos MRC, Zellerkraut H, Oliveira LR. Curso de orientação à gestação: repercussöes

nos pais que vivenciam o primeiro ciclo gravídico. Mundo Saúde. 2008;32(4):420-9.

11. Ministério da Saúde (BR). Pré-natal e puerpério. Brasilia: Ministério da Saúde; 2005.

Tomeleri KR, Pieri FM, Violin MR, Serafim D, Marcon SS. “Eu vi meu filho nascer”: vivência

dos pais na sala de parto. Rev Gaúcha Enferm. 2007;28(4):497-504.

12. Carvalho CCM, Souza ASR, Filho OBM. Prevalência e fatores associados

à prática da episiotomia em maternidade escola do Recife, Pernambuco, Brasil. AMB

Rev Assoc Med Bras. 2010;56(3):333-9.

13. Rezende J, Montenegro CAB. Obstetrícia fundamental. $10^{\mathrm{a}}$ ed. Rio de Janeiro:

Guanabara Koogan; 2006.

14. Soares RK, Silva SF, Lessa PR, Moura ER, Pinheiro PN, Damasceno AK. Parturient's

companion and their relationship with the nursing team: a qualitative study. Online

Braz J Nurs (Online). 2010;17(9).

15. Soares RK, Silva SF, Lessa PR, Moura ER, Pinheiro PN, Damasceno AK. Parturient's

companion and their relationship with the nursing team: a qualitative study. Online

Brazilian Journal of Nursing. 2010. June 17;9 (1). 